\title{
Distributed electric current and its relation to ultraviolet radiation of the active region
}

\author{
Yu.A. Fursyak, A.S. Kutsenko \\ Crimean Astrophysical Observatory, Nauchny, Crimea, 298409 \\ e-mail: yuriy_fursyak@mail.ru
}

Submitted on July 7, 2020

\begin{abstract}
We used the magnetographic data of the Helioseismic and Magnetic Imager (HMI) instrument onboard the Solar Dynamics Observatory (SDO) to calculate vertical electric currents in the NOAA active region (AR) 12192. The AR was tracked between October 22, 2014 and October 25, 2014 with 720 s cadence. We revealed the presence of a large-scale electric current structure - distributed electric current - with the absolute magnitude varying in the range of (40-90) $\cdot 10^{12} \mathrm{~A}$. The distributed electric current is supposed to exist throughout the entire AR, and, extending to the upper layers of the solar atmosphere in one part of the AR, it closes through the chromosphere and corona in the remaining part of the AR. To test this assumption, we have compared the temporal variation of the distributed electric current value with the flare activity level (using GOES-15 data), as well as with intensity of ultraviolet radiation (UV) in the AR (using the Atmospheric Imaging Assembly (AIA/SDO) data in channels $94 \AA$, $193 \AA, 304 \AA$, and $1600 \AA$ ). We found that: i) Time intervals of enhanced flare activity are co-temporal with intervals of increased values of the distributed electric current. The absence of rapid changes in the value of the distributed electric current during solar flares can be explained by high inductance of the current-carrying magnetic loops. ii) Rough estimates of the magnetic energy carried by the distributed electric current into the corona yield the values of about $10^{33}-10^{34}$ erg for 12192 . Only a small amount of this energy is released during flare processes in the AR. Most of this energy seems to be consumed during other dissipative processes in the corona. iii) Comparison of the temporal variations of intensity in the $193 \AA$ UV-radiation channel with dynamics of the distributed electric current in the AR reveals a good positive correlation between these values (Pearson's $\mathrm{R}=0.63$ ). The absence of a correlation between the distributed electric current value and the intensity of UV radiation in channels $1600 \AA, 304 \AA$ and $94 \AA$ might be explained by a low efficiency of the coronal loop heating by ohmic dissipation of electric currents in the corona due to the strong dependence of plasma conductivity on temperature. iv) Our results may support the concept of equivalent LRC circuit of a current-carrying coronal magnetic loop proposed by Alfven and Carlqvist in 1967 and developed by V.V. Zaitsev, A.V. Stepanov, and others. According to this model, the large-scale electric currents must exist in the upper layers of the solar atmosphere and take part in the coronal plasma heating.
\end{abstract}

Key words: Sun, active regions, solar activity, electric currents, coronal heating

\section{Introduction}

Despite the large arrays of information (daily data of just the Atmospheric Imaging Assembly (AIA, Lemen et al., 2012) instrument onboard the Solar Dynamics Observatory (SDO) account for about 2 terabytes), the presence of modern ground-based and space telescopes (for example, SOHO, Hinode, TRACE, SDO, and others), the problem of the solar corona heating remains one of unresolved ones in modern solar physics.

Different authors distinguish a number of heating mechanisms of coronal plasma which can be divided into two large groups - external (free magnetic energy comes from outside) and internal (the plasma heating is implemented due to the conversion of free energy of a magnetic loop). To the external heating mechanisms one can refer the evaporation of chromospheric plasma into the corona (Antonucci et al., 1998; Bornmann, 1998), dissipation of Alfven waves (Hollweg, 1984; Ionson, 1984), cyclotron absorption of electromagnetic waves generated during a flare (Melrose, Dulk, 1984), and others. Among internal heating mechanisms of coronal plasma one can distinguish the microflares caused by reconnections inside a magnetic loop (Parker, 1988), tearing instability (Galeev et al., 1981), and ohmic dissipation of electric currents (Spicer, 1991).

The accumulation of electric currents in the corona can be implemented by two ways. The first one implies the formation of electric currents at the photospheric level as a result of the twisting or shifting of a magnetic tube (McClymont, Fisher, 1989; Török, Kliem, 2003; 
Aulanier et al., 2005; Dalmasse et al., 2015). The second mechanism implies the twisting of a magnetic tube in the depth of the convection zone, at the stage of its formation or rise (Cheung, Isobe, 2014), which leads to a buoyancy of the magnetic flux with already formed system of electric currents (Leka et al., 1996; Longcope, Welsch, 2000).

The existence of electric currents in the solar atmosphere over long time intervals suggests the electric circuit with current to be closed. A number of theoretical models (Parker, 1996; Aulanier et al., 2005; Török et al., 2014; Dalmasse et al., 2015) predict a closing of such circuit at the photospheric level and the presence of both direct and inverse electric currents in a magnetic tube (electric currents in such a magnetic tube should be compensated). However, observational data indicate that electric currents in individual polarities of the magnetic field of an active region (AR) in most cases are not fully compensated (Georgoulis et al., 2012). This especially concerns the regions with high flare productivity (Liu et al., 2017; Kontogiannis et al., 2017). Moreover, the detection of inverse currents in an AR is a complicated task, and one can currently find very few studies having evidence of their existence (see, e.g., Gosain et al., 2014). Melrose (1991) explains the complexity of inverse current detection by either very low absolute values (lower than the detection limit) of these currents, which are also distributed over a large area in an AR, or the fact that these currents are concentrated at small unresolved areas. An alternative explanation to be considered in detail is that inverse currents can reside lower than the photospheric level (see Fig. 3 in Melrose, 1995).

The idea to represent a current-carrying loop as an analog of the closed electric circuit with a closing of currents under the photosphere was formulated in 1967 (Alfven, Carlqvist, 1967). The concept was based on the first measurements of vertical electric currents in the photosphere carried out by A.B. Severny (Severny, 1964). Further, the assumption that electric currents can close lower than the photospheric level can be found in Abramenko et al. (1991), and namely the model of equivalent LRC circuit for the description of the processes in a current-carrying coronal loop was developed in the studies of V.V. Zaitsev, A.V. Stepanov, and other authors (Zaitsev, Khodachenko, 1997; Zaitsev et al., 1998; Zaitsev, Stepanov, 2008; Stepanov et al., 2012; Zaitsev, Kronshtadtov, 2016). Following this model, electric currents are generated by convective plasma motions at the photospheric level or, in the case of locating the base of magnetic loops in the region of sunspots, by rotational motions of sunspots with a velocity of about $0.1 \mathrm{~km} \mathrm{~s}^{-1}$ (Stenflo, 1969). The electric current generated at the photospheric level flows through the chromosphere and the corona from one loop base to the other one. Since, as shown above, distinct manifestations of inverse electric currents have not been detected, the inverse electric current is assumed to flow below the photosphere by the shortest way from one loop base to the other one closing the circuit.

The calculations performed within the circuit model of a current-carrying magnetic tube show that convective motions in the photosphere with a velocity of about $0.1 \mathrm{~km} \mathrm{~s}^{-1}$ result in generating thin magnetic tubes with a radius of 100$1000 \mathrm{~km}$ and electric current inside of about $10^{11}-10^{12} \mathrm{~A}$ (Zaitsev, Khodachenko, 1997; Khodachenko, Zaitsev, 2002). These calculations are in good agreement with the results presented in Zaitsev et al. (1998), in which on the basis of observational data derived at the Metsahovi Radio Research Station in the millimeter wavelength range the values of electric currents $10^{11}-1.42 \cdot 10^{12}$ A were obtained for 16 flare events, and also with a number of other observational data.

Since the large-scale electric currents generated in the depth of the convection zone or at the photospheric level flow high in the corona, then there should be observed different processes and phenomena associated with dissipation of these currents in upper layers of the solar atmosphere. A spectroscopic analysis of coronal magnetic loops performed in the late 20th century using instruments onboard the spacecraft SOHO made it possible to detect only insignificant variations in temperature of coronal loops with height (Aschwanden et al., 1999, 2000). Analogous results were acquired from the analysis of observational data derived with the spacecraft TRACE (Reale, Peres, 2000). A small temperature gradient in the coronal region of a magnetic loop means that the source of heating should be in thermal balance with radiative losses because otherwise it is impossible to ensure the observed quasistationarity of a magnetic loop over a large time (at least for several hours). The calculations performed in Zaitsev, Shibasaki (2005) show that a possible heating mechanism of coronal loops is ohmic dissipation of electric currents. The coronal plasma heating and the acceleration of particles in the magnetic arch are associated with Cowling conductivity (Cowling, 1957) caused by collisions of ions with neutral atoms. The presence in the corona of even a small number (of about $10^{-5}$ of the total mass) of neutral helium atoms provides a growth of resistivity of the magnetic loop by 8-9 orders, up to anomalous values (Holman, 1985; Sprangler, 2009) required for the effective heating of coronal plasma due to ohmic dissipation of distributed electric currents.

In the previous study (Fursyak et al., 2020), we have detected the distributed electric current $\left(I_{\text {distr }}\right)$ in six active regions of Solar Cycle 24, which were different in magnetic, morphological parameters, and the flare productivity level. We assumed that the distributed electric current extends through the entire AR: comes into the upper layers of the atmosphere in one its part and closes through the chromosphere and the corona in the remaining part. If it is true, then we should observe a link between temporal variations in the value of the distributed electric current and processes in the solar corona. A search for the presence of such a dependence and the establishment of a character of interaction between variations in the value of the distributed electric current and possible dissipative processes in the corona on the example of the large flare-producing NOAA AR 12192 is the main objective of the study.

\section{A target and observational data}

The target - NOAA AR 12192 (Fig. 1a) - is the largest in area and one of the most active sunspot groups of Solar Cycle 24 (Sun et al., 2015; Chen et al., 2015; Thalmann et al., 2015; McMaken, Petrie, 2017; Jain et al., 2017). Monitoring of the region was started on October 22, 2014 at 00:00 UT. The end of the monitoring - October 25, 2014 at 23:59 UT. Over the time of observations the region under investigation was within $\pm 30^{\circ}$ in longitude with respect to the central merid- 

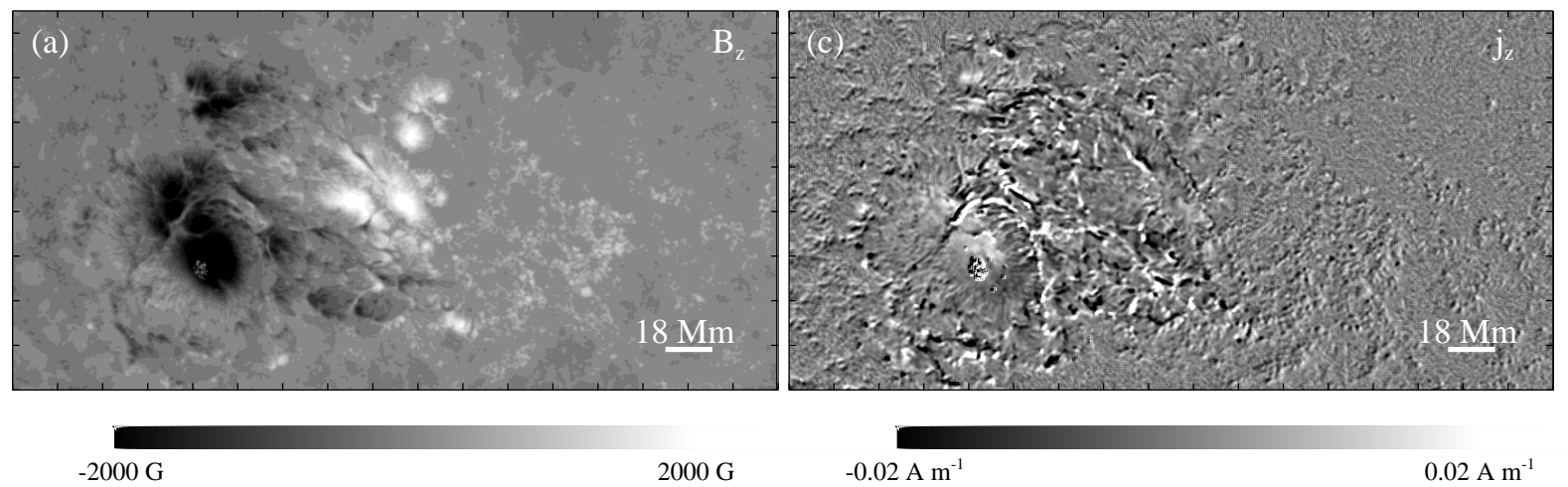

$-0.02 \mathrm{~A} \mathrm{~m}^{-1}$ $0.02 \mathrm{~A} \mathrm{~m}^{-1}$
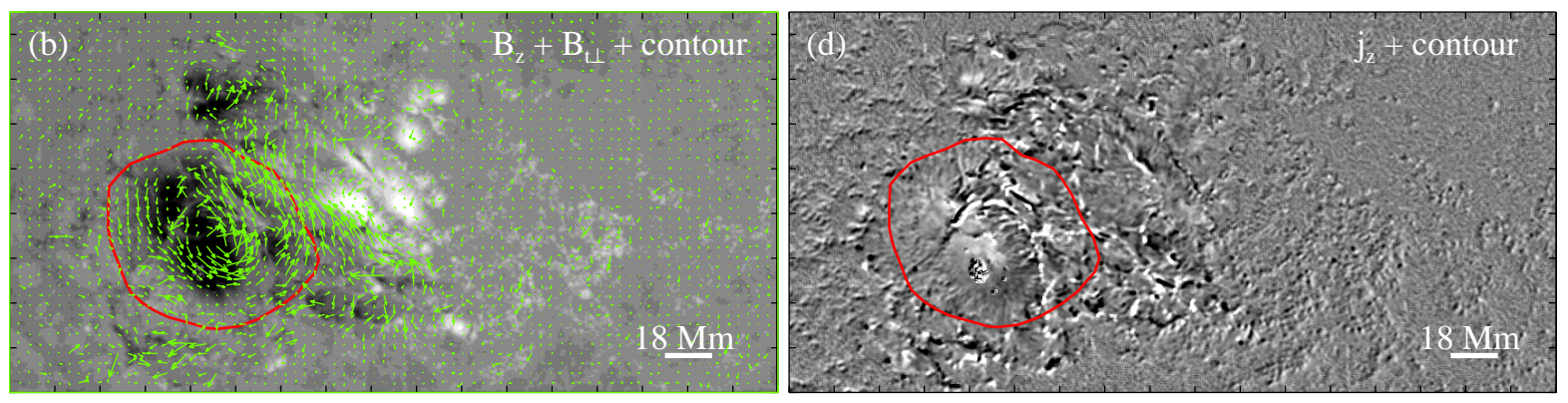

Fig. 1. (a) Longitudinal magnetogram $B_{Z}$ for the NOAA AR 12192 taken on October 22, 2014 at 00:00 UT with HMI/SDO. The map is scaled from $-2000 \mathrm{G}$ (black) to $2000 \mathrm{G}$ (white), the corresponding scale is given at the bottom of the image. (b) the $B_{\mathrm{Z}}$ component of the magnetic field (background) with superimposed vector azimuths of the nonpotential component of the horizontal magnetic field of the region $\boldsymbol{B}_{\mathrm{t} \perp}$ (green arrows). The arrow length is proportional to the value of the nonpotential field component. The red closed curve around

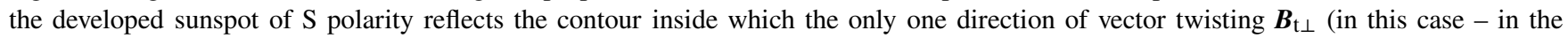
anti-clockwise direction) prevails. Inside of the given contour, according to the map of vertical electric current density (see (d)), the value of distributed electric current was calculated (more details can be found in the text and in Fursyak et al., 2020). (c) The map of vertical electric current density for the NOAA AR 12192 on October 22, 2014 at 00:00 UT calculated on the basis of data on the magnetic field horizontal components of the region. The map is scaled from $-0.02 \mathrm{~A} \mathrm{~m}^{-2}$ (black) to $0.02 \mathrm{~A} \mathrm{~m}^{-2}$ (white), the corresponding scale is given at the bottom of the image. In all the images, north is at the top, west is to the right

ian, which allowed us to minimize errors in calculations of vertical electric currents and distributed electric current that arise due to the projection effect.

The initial data for calculating electric currents are the Spaceweather HMI Active Region Patch (SHARP, Bobra et al., 2014; Hoeksema et al., 2014) magnetograms of magnetic field vector components for the studied AR available on the website of the Joint Science Operations Center $(\text { JSOC })^{1}$ (series hmi.sharp_720s, 12 min cadence). Magnetograms were acquired by the Helioseismic and Magnetic Imager (Scherrer et al., 2012) onboard the Solar Dynamics Observatory (Pesnell et al., 2012).

The data for calculating the total intensity of ultraviolet radiation for the AR are photoheliograms acquired by the AIA (Lemen et al., 2012) instrument onboard SDO and available on the mentioned website of JSOC. We have used solar photoheliograms in four wavelength ranges of ultraviolet radiation: $94 \AA, 193 \AA$, $304 \AA$ (series aia.lev1_euv_12s, data with 12 s cadence) and $1600 \AA$ (series aia.lev1_uv_24s, data with 24 s cadence).

To estimate the flare activity level, we have used the GOES-15 (Geostationary Operational Environmental Satel-

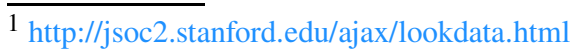

lite $)^{2}$ data on the X-ray emission flow in the range of $1-8 \AA$ in Earth's orbit.

\section{Basics of the calculation technique for vertical electric currents, distributed electric current, and intensity of ultraviolet radiation in the $\mathbf{A R}$}

The calculation of vertical electric currents was carried out by the integral method (Abramenko, Gopasyuk, 1987; Fursyak, 2018) on the basis of magnetograms of vector components of the transverse magnetic field of the AR:

$$
\left(I_{z}\right)_{i, j}=\frac{1}{\mu_{0}} \int_{L} \boldsymbol{B}_{t} d r
$$

where $\mu_{0}=4 \pi \cdot 10^{-7} \mathrm{G} \mathrm{m}^{-1}$ is the magnetic permittivity constant; $\boldsymbol{B}_{t} \equiv\left(B_{x}, B_{y}\right)$ - the transverse magnetic field vector of the AR; $d r$ - the element of integration, which is equal to the pixel size in the HMI/SDO magnetogram $(\sim 363 \mathrm{~km})$;

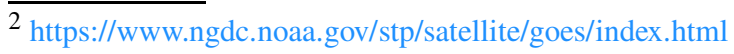


Table 1. Basic characteristics of four UV radiation channels under analysis

\begin{tabular}{rccc}
\hline AIA/SDO channel & Source & Characteristic temperature & Solar atmosphere region \\
\hline $1600 \AA$ & C IV, continuum & $100000 \mathrm{~K}, 5000 \mathrm{~K}$ & Transition region, upper photosphere \\
$304 \AA$ & He II & $50000 \mathrm{~K}$ & Chromosphere and transition region \\
$193 \AA$ & Fe XII & $1200000 \mathrm{~K}$ & Corona \\
$94 \AA$ & Fe XVIII & $6300000 \mathrm{~K}$ & Flare regions in the corona \\
\hline
\end{tabular}

$i, j$-coordinates of the pixel in the magnetogram for which the electric current is calculated.

The integral on the right side of Expression (1) was calculated approximately using Simpson's method. The $L$ contour had a rectangular shape and the $5 \times 5$ pixel size. While integrating, the contour cutoff was in the anti-clockwise direction. Thus, we obtained the integral current values through the $L$ contour. The averaging of current over the contour area yields us the estimate of vertical current density in the central pixel of this contour. Calculating the values of vertical current for each pixel of the SHARP magnetogram, there were constructed the maps of vertical electric current density in the AR at a given moment of time (Fig. 1c).

To detect the distributed electric current in the AR, the indirect technique was used that is described in detail in Fursyak et al. (2020) since the maps of vertical electric current density do not allow us to reveal the presence of largescale electric current structures distributed over large areas. From the physical point of view, the method is quite simple and its sense can be reduced to the fact that electric current generates a vortex magnetic field in the plane that is perpendicular to the electric current itself, and this field can be detected. Comparing the observed horizontal magnetic field with the calculated potential transverse magnetic field of the $\mathrm{AR}$ and singling out a component that is orthogonal to the direction of the potential field vector, we obtain a map of distribution of the nonpotential transverse field component $\left(\boldsymbol{B}_{\mathrm{t} \perp}\right)$ in the AR. In such maps, one can clearly see the regular vortex structures of the vector $\boldsymbol{B}_{\mathrm{t} \perp}$ near large sunspots caused by the presence of distributed electric currents (Fig. 1b). If we outline the $C$ (the red closed curve in Fig. 1b) contour around the sunspot in such a way that inside the contour the direction of twisting of the nonpotential transverse field component has one prevailing direction, and, using the map of currents, calculate the resultant electric current inside such a contour (Fig. 1, d), then we obtain the value of distributed electric current $I_{\mathrm{distr}}$ at this point in time:

$I_{\mathrm{distr}}=\sum_{\mathrm{C}} j_{i, j} \cdot \Delta s$.

To calculate the total intensity of ultraviolet radiation of the AR in different ranges, the appropriate AIA/SDO photoheliograms were used. These photoheliograms represent images of the full solar disk with a size of $4096 \times 4096$ pixels. To calculate the intensity of UV radiation above the studied AR, we used the image cropping available on the website of JSOC. Thus, we obtained a patch which approximately corresponded in size to the sizes of SHARP magnetograms of the magnetic field vector for the AR. The total intensity of UV radiation in a certain channel was calculated by a simple addition of intensities of radiation in each pixel of the patch:
$E_{\lambda}=\sum E_{i, j}$

We performed a calculation of the ultraviolet radiation intensity in four wavelength ranges of UV radiation - $1600 \AA$, $304 \AA, 193 \AA$, and $94 \AA$. The basic characteristics of the chosen ranges are given in Table 1 .

Comparison of data on the dynamics of the distributed electric current with UV radiation intensity for the AR in different wavelength ranges is the main objective of our research. But before comparing data, they need to be attributed to the same time interval and the same region on the Sun. The main problem is that the AIA/SDO instrument yields information with $12 / 24 \mathrm{~s}$ cadence, whereas HMI/SDO - with $720 \mathrm{~s}$ cadence (12 minutes). From the initial time array we selected only those photoheliograms that had been derived at the moments of time close as much as possible to the time of deriving magnetograms of magnetic field vector components with HMI/SDO.

The selected AIA/SDO data were further filtered. The aim of this filtration is to eliminate incorrect measurements. From the array of information we have discarded the data derived every day at 18:00 UT (at this time a calibration of SDO instruments takes place, and the AIA instrument provides values of resultant radiation intensity close to zero). Particular attention was paid to the data derived with AIA/SDO during flares. Since during flare processes the intensive radiation in ultraviolet is observed, the dynamic range in some channels of AIA/SDO turns out to be insufficient to adequately reflect the radiation level and the instrument starts operating in the other mode. The distorted data were not also taken into account. Thus, to construct the diagrams of time dependences, as well as the correlation diagrams, only those data from AIA/SDO were used that were free of any distortions associated with artifacts of the instrument itself and at the same time coinciding in time with the data derived with HMI/SDO.

In our case from four UV radiation ranges selected for analysis the greatest number of distortions was observed in the $193 \AA$ A channel. The data derived in other channels were adduced to the unified system based on the $193 \AA$ channel. For that reason, a significant intermittency of information on the UV radiation intensity is observed in all four channels (see Fig. 3).

\section{Results}

In the previous study (Fursyak et al., 2020), we showed the existence of large-scale electric current structures in the AR. A low (significantly less than $1 \%$ ) unbalance of vertical electric currents calculated over the entire AR made it possible to 


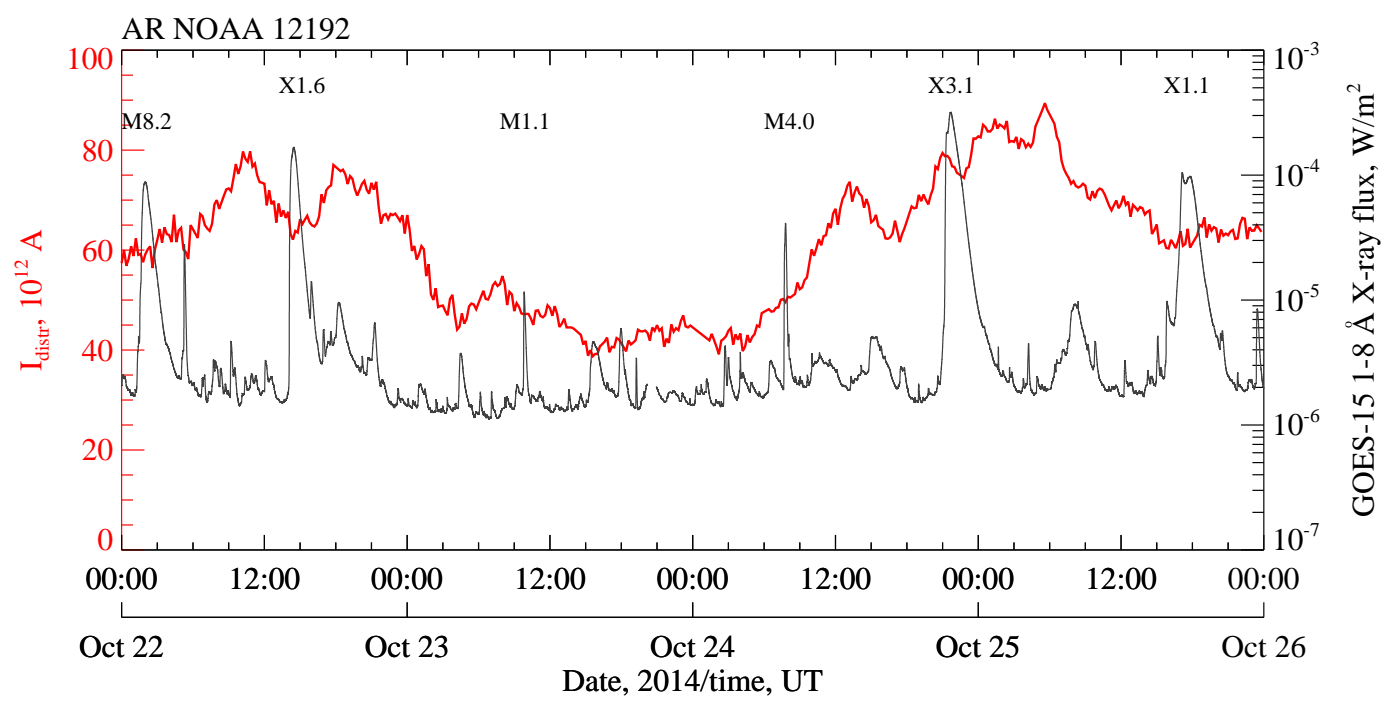

Fig. 2. Dynamics of the distributed electric current in the NOAA AR 12192 over the time of monitoring (red curve). The grey line denote temporal variations in the X-ray radiation in a wavelength range of 1-8 $\AA$ in Earth's orbit (data from the spacecraft GOES-15). The X-ray classes of the strongest flares associated with the NOAA AR 12192 are given

assume that the distributed electric current flows to the upper layers of the solar atmosphere in one part of the AR closing through the chromosphere and the corona in the rest part of the AR. If it is true, then the calculated electric current, carrying a large amount of free magnetic energy into the upper layers of the solar atmosphere, should be associated with flares and other dissipative processes in the corona. In the same study, on the example of 6 ARs there was analyzed a relation between temporal variations of the distributed electric current and the flare activity level. The absolute magnitudes of the distributed electric current and the amplitude of its variations were shown to be higher in the regions with the higher level of flare productivity.

Here we consider the distributed electric current and its dynamics in the NOAA AR 12192. As it follows from Fig. 1a, c, the most developed vortex structure of the nonpotential component of the horizontal magnetic field vector caused by the presence of the large-scale electric field was detected around a large sunspot of S polarity in the following part of the AR. According to the direction of vector twisting $\boldsymbol{B}_{\mathrm{t} \perp}$ (in the anti-clockwise direction) we established that the sign of the distributed electric current should be positive (current is directed upward, toward an observer), and, consequently, the present electric current structure is not associated with the developed sunspots of $\mathrm{N}$ polarity to the west of the considered sunspot (in which the vector twisting $\boldsymbol{B}_{\mathrm{t} \perp}$ also implies the presence of the positive electric current) but with an extensive region of weak magnetic fields of $\mathrm{N}$ polarity in the south-west. Our assumption is in good agreement with the data of theoretical calculations. Thus, Jiang et al. (2016) show the presence of the large-scale electric current structure flowing to the corona at a height of about $46 \mathrm{Mm}$ and associated, on the one hand, with the large sunspot of S polarity and, on the other hand, with the region of weak magnetic fields of $\mathrm{N}$ polarity to the south-west of the large sunspot.

We analyzed time variations of the calculated distributed electric current and compared them with the flares which had been produced by the studied AR during the monitoring time (Fig. 2). The diagram shows that the strongest flares observed in the AR during October 22, 2014, as well as October 2425,2014 , can be associated with the local maxima of $I_{\text {distr }}$. During the time interval between October 23 - the first half of October 24 the distributed electric current in the region reached its minimum for all the time of monitoring (of about $40 \cdot 10^{12} \mathrm{~A}$ ). At the same time interval, no flares stronger than M5.0 were observed.

The high values of distributed electric current indicate a large supply of free magnetic energy carrying to the upper layers of the solar atmosphere. Thus, Abramenko, Gopasyuk (1987) carried out an approximate estimate of energy carrying to the corona by the global (large-scale) electric current of about $10^{12} \mathrm{~A}$. The values of about $10^{32} \mathrm{erg}$ were derived which are comparable with the total energy released during a strong solar flare. In our recent study (Fursyak et al., 2020), even in an AR with low flare activity the presence of distributed electric currents of $\pm(10-20) \cdot 10^{12}$ A was revealed, which (according to rough estimates) corresponds to the energy of $10^{32}-10^{33} \mathrm{erg}$. The minimum values of the distributed electric current in the NOAA AR 12192, as can be seen in Fig. 2, are close to the values of $40 \cdot 10^{12} \mathrm{~A}$, which is equivalent to the energy of $10^{33}-10^{34} \mathrm{erg}$. Despite such a huge supply of magnetic energy in the corona, strong flares were not observed within time interval. This means that a great part of energy carried by electric currents to the corona is manifested not as flares but through other dissipative processes, and only a small amount of energy is released in flares. The present assumption is confirmed by earlier studies of other authors (Zaitsev et al., 1998; Zaitsev, Stepanov, 2008), who suggest that not more than 5-10\% of energy accumulated in electric current is released during a solar flare. One of the most probable mechanisms of current energy dissipation in the corona not associated with flare energy release can be a heating of coronal plasma by electric currents flowing in the upper layers of the solar atmosphere. 

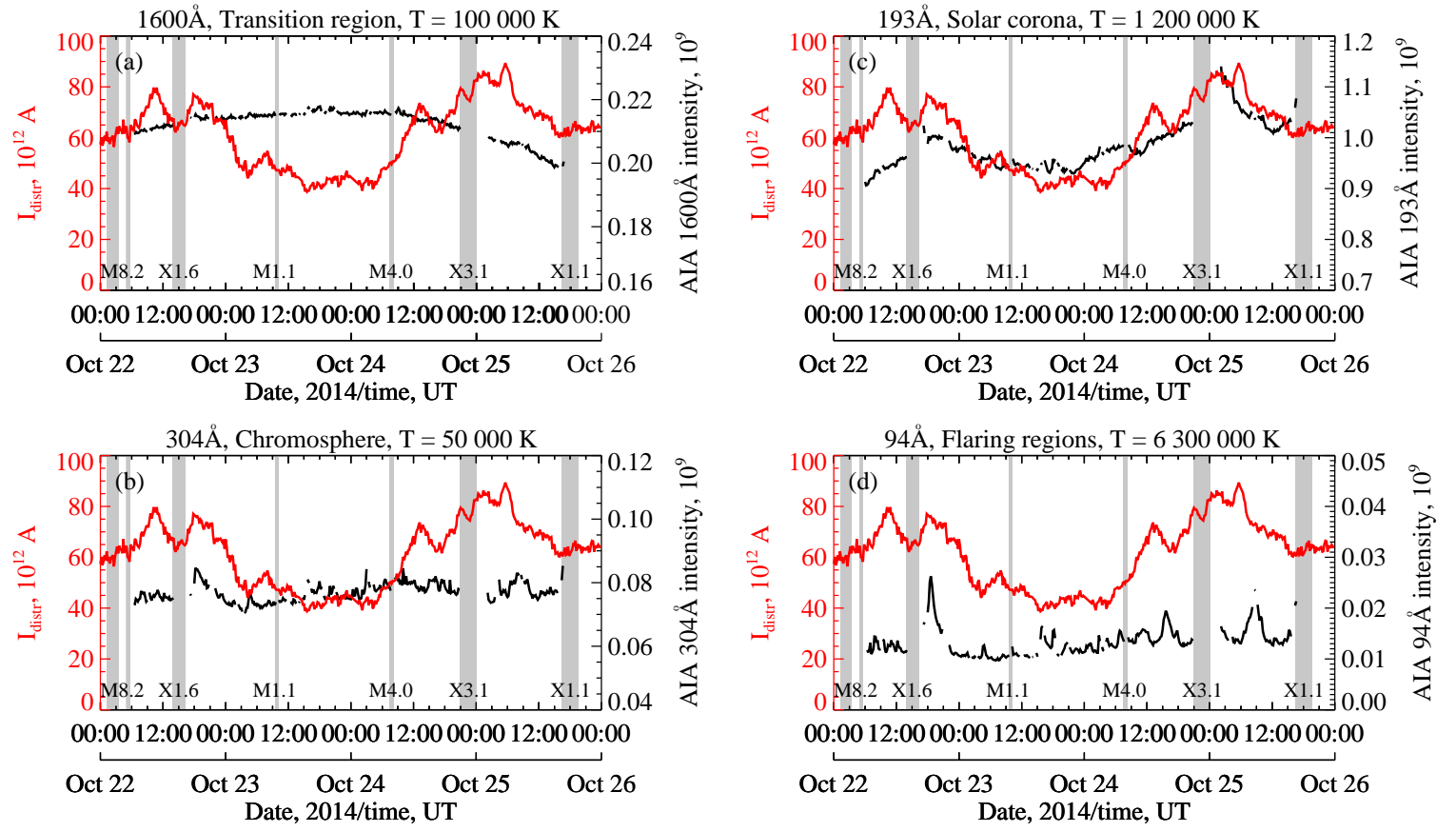

Fig. 3. Time variations in the distributed electric current (red curve) and total intensity of ultraviolet radiation in the AR (black curve) in channels $1600 \AA$ (a), $304 \AA$ (b), $193 \AA$ (c), $94 \AA$ (d). Data on the intensity of UV radiation were derived with AIA/SDO. A high intermittency of the derived information is caused by peculiarities of the instrument operation (see the text for more details). Grey vertical stripes denote time intervals throughout which the X-ray radiation flow from the AR under investigation in the range of 1-8 $\AA$ (according to the GOES-15 data) exceeded a value of $10^{-5} \mathrm{~W} \mathrm{~m}^{-2}$ (the M1.0 class X-ray flare equivalent). The strongest flare processes associated with the studied region are indicated at the bottom of diagrams

A study of the relation between temporal variations in the distributed electric current and the flare activity level presented in Fig. 2 allows us to make two important conclusions. In particular, it should be noted a more gradual character of temporal variations in the distributed electric current as compared to the X-ray radiation flow. One can interpret this by the fact that the distributed electric current is a large-scale structure which has existed over a significant time interval covering both the entire AR and all layers of the solar atmosphere. Meanwhile, the flare is a phenomenon that is less substantial in both spatial and time intervals.

Fig. 2 shows the absence of dramatic variations in the distributed electric current during flares. Meanwhile, many models suggest a direct participation of electric currents and electric current sheets in the flare process (Alfven, Carlqvist, 1967; Parker, 1973; Sokolov, Kosovichev, 1978; Heyvaerts et al., 1977; Priest, Forbes, 2002; Sui, Holman, 2003; Li et al., 2018). This contradiction can be explained by high induction of a current-carrying loop (Alfven, Carlqvist, 1967; Khodachenko et al., 2003) and, consequently, by a slow response of the system of large-scale electric currents to the events in the upper layers of the solar atmosphere.

Fig. 3 displays the curves of variations in $I_{\text {distr }}$ and UV radiation intensity in four channels of the AIA/SDO instrument.

To check the validity of our assumption that the distributed electric current flows to the upper layers of the solar atmosphere and can take part in coronal plasma heating, we compared temporal variations of $I_{\text {distr }}$ and UV radiation intensity (Fig. 3) in four wavelength ranges (see Table 1) stratified in temperature and, accordingly, in height. The correlation diagrams were also acquired, which reflect the character of interaction between $I_{\text {distr }}$ and UV radiation intensity in each of the considered ranges (Fig. 4).

It can be seen in diagrams presented in Fig. 3 that for channels $1600 \AA$ and $304 \AA$ the temporal variations in the distributed electric current are not associated with variations in UV radiation intensity. Thus, the variation in UV radiation intensity in the $1600 \AA$ channel can be explained by the effect of limb darkening. A weak interaction between the distributed electric current and UV radiation intensity in the $304 \AA$ channel is confirmed by correlation diagrams: for the range of $304 \AA$ (Fig. 4b) the correlation coefficient equals 0.22 .

Despite the absence of a distinct relation between the distributed electric current and UV radiation intensity in the $94 \AA$ channel (as it follows from Fig. 4d, $k=0.45$ ), the diagram of temporal variations of the studied values (Fig. 3d) allows us to observe insignificant in amplitude chaotic variations in radiation intensity not associated with flare processes. Moreover, the most significant peaks of UV radiation intensity are close in time to the local maxima of the distributed electric current lagging behind in a time scale of 1-4 hours. This observation can be a signature of not only involving electric currents in dissipative processes in the upper layers of 

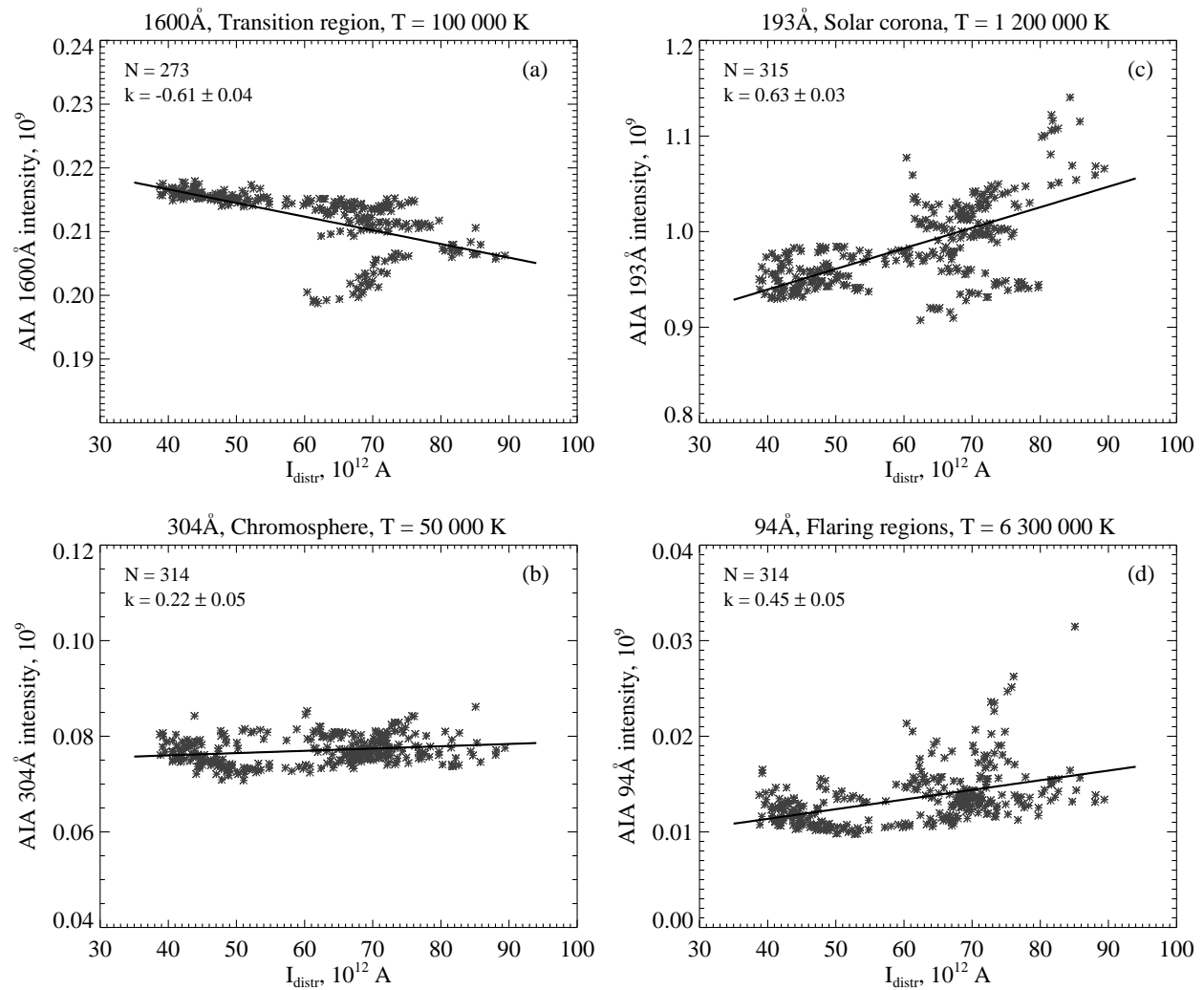

Fig. 4. Diagrams of the correlation between the distributed electric current (horizontal scale) and total UV radiation intensity (vertical scale) in channels $1600 \AA$ (a), $304 \AA$ (b), $193 \AA$ (c), $94 \AA$ (d). The correlation coefficients in each case are indicated in the upper left corner of the corresponding diagrams

the solar atmosphere but the correctness of the proposed assumption on high inductance of a magnetic current-carrying tube.

The other pattern is observed for the $193 \AA$ channel (Fig. 3c). Despite a high intermittency of data, we can note two maxima of UV radiation intensity in a given range. One of them was observed throughout October 22, 2014, the second - October 24-25, 2014. The maxima of UV radiation intensity in the $193 \AA$ channel coincide in time with the maxima of $I_{\text {distr. }}$. The diagram in Fig. 4c shows the presence of good correlation dependence between the analyzed values (the correlation coefficient $k=0.63$ ).

An analysis of temporal variations in the distributed electric current and UV radiation intensity in four different ranges (Fig. 3), as well as correlation dependences between these values allow us to suggest that the distributed electric current can play a significant role in heating coronal loops. Unfortunately, there is no unambiguous simple association between the temperature of plasma in the corona and the UV radiation intensity in individual spectral lines. In the general case, the problem of measuring temperature in the corona requires to solve a problem on the inversion of the differential emission measure for a series of spectral lines (e.g., Morgan and Pickering, 2019). Nonetheless, the observed good correlation (Fig 4c) between the distributed electric current and UV radiation intensity in the $193 \AA$ channel (Fig. 3c) can evidence for the involvement of ohmic current dissipa- tion to the energy release in ultraviolet. If the assumption is true, this result is in good agreement with the concept of the equivalent LRC-circuit of a current-carrying magnetic loop considered in Zaitsev, Khodachenko (1997), Zaitsev et al. (1998), Zaitsev, Stepanov (2008), Stepanov et al. (2012), Zaitsev, Kronshtadtov (2016). According to this model, the large-scale electric currents should exist in the corona and be involved in coronal plasma heating.

The absence of a distinct relation between temporal variations in UV radiation intensity in other channels and $I_{\text {distr }}$ (Fig. 4a, b, d) can indicate that the coronal plasma heating due to ohmic dissipation of electric currents is effective only in a certain temperature range. This is associated with plasma conductivity, which strongly depends on temperature $\left(\sigma T^{3 / 2}\right)$. At low (typical of the chromosphere and the transition layer) and high (hot flare plasma, high-temperature plasma in the corona) temperatures, the effectiveness of heating coronal structures due to ohmic dissipation of currents significantly decreases.

Note that, since the energy accumulated in electric current is proportional to its square $\left(W_{\mathrm{m}} \sim I^{2}\right)$, we also considered the correlation dependence between UV radiation intensities in the ranges of $1600 \AA, 304 \AA, 193 \AA, 94 \AA$ and the square value of $I_{\text {distr }}$. However, these variations did not lead to the expected results: correlation changed by a few percent. For instance, the dependence $I_{\text {distr }}^{2}$ vs $E_{193 \AA}$ showed the correlation of $k=0.65$, whereas the correlation coefficient for 
the dependence $I_{\text {distr }}$ vs $E_{193 \AA}$ accounted for 0.63 (Fig. 4c). Such a result allows one to suppose that flares and ohmic dissipation seem to be not the only processes the distributed electric currents in the corona to be involved into.

\section{Conclusions and discussion}

The detection of distributed electric current and the comparison of its dynamics with a variation in the flare productivity level of the region and temporal variations of UV radiation in different wavelength ranges on the example of the NOAA AR 12192 allow us to make the following conclusions:

1. The presence of a correlation $(k=0.63)$ between the value of distributed electric current $I_{\text {distr }}$ and UV radiation intensity in the AIA/SDO $193 \AA$ channel can be a possible indication that the detected distributed electric current indeed flows into the corona. Moreover, the ohmic dissipation of large-scale currents, along with other processes, can be one of important mechanisms of coronal loop heating.

2. A rough estimate shows that the distributed electric current releases energy of about $10^{33}-10^{34} \mathrm{erg}$ into the upper layers of the solar atmosphere, which significantly exceeds the total energy of even strong flares. A large part of energy of large-scale electric currents seems to be implemented as other dissipative processes in the corona.

3. The absence of dramatic variations in the value of distributed electric current during strong flare processes, as well as the presence of small-amplitude peaks of UV radiation intensity in the $94 \AA$ channel associated with local maxima of distributed current take place due to high inductance of a magnetic current-carrying tube.

4. The absence of a correlation between the dynamics of distributed electric current and UV radiation in $1600 \AA$, $304 \AA$ channels and a weak correlation in the $94 \AA$ channel can be explained by a strong dependence of plasma conductivity on temperature. A certain contribution into the heating of coronal structures can be made by ohmic dissipation of electric currents only at the temperatures characteristic of the corona (of about $0.5-2.5 \mathrm{MK}$ ). At lower or higher temperatures (for example, in the hot flare plasma) this mechanism loses its effectiveness and the coronal plasma heating occurs due to other processes.

The absence of noticeable improvement in the correlation dependence between the squared value of distributed electric current and radiation intensities in different wavelength ranges of UV radiation as compared to the results presented in Fig. 3 and Fig. 4 allows us to suggest that, apparently, the solar flares and ohmic dissipation of electric currents are not the only processes that involve distributed electric currents in the upper layers of the solar atmosphere.

Acknowledgements. The study of distributed electric currents and their relation to the flare productivity of the AR was supported by the Russian Science Foundation (RSF) \# 18-12-00131; the analysis of temporal variations in UV radiation intensities in the corona above the studied AR was performed within the research work proceeding at the Department of Physics of the Sun and the Solar System of CrAO
RAS \# 0831-2019-0006 "The general and local characteristics of the Sun". Authors are grateful to the referee for the interest in the paper and useful comments which helped to improve the manuscript.

\section{References}

Abramenko V.I., Gopasyuk S.I., 1987. Izv. Krymsk. Astrofiz. Observ., vol. 76, pp. 147-168. (In Russ.)

Abramenko V.I., Gopasyuk S.I., Ogir' M.B., 1991. Izv. Krymsk. Astrofiz. Observ., vol. 83, pp. 3-11. (In Russ.)

Alfven H., Carlqvist P., 1967. Solar Phys., vol. 1, pp. 220228.

Antonucci E., Alexander D., Culhane J.L., et al., 1998. In Strong K.T., et al. (Eds), The Many Faces of the Sun: A Summary of the Results from NASA's Solar Maximum Mission. Berlin: Springer, p. 331.

Aschwanden M.J., Alexander D., Hurlburt N., et al., 2000. Astrophys. J., vol. 531, pp. 1129-1149.

Aschwanden M.J., Newmark J.S., Delabourdiniere J.-P., et al., 1999. Astrophys. J., vol. 515, pp. 842-867.

Aulanier G., Demoulin P., Grappin R., 2005. Astron. Astrophys., vol. 430, pp. 1067-1087.

Bobra M.G., Sun X., Hoeksema J.T., et al., 2014. Solar Phys., vol. 289, pp. 3549-3578.

Bornmann P.L., 1998. In Strong K.T., et al. (Eds), The Many Faces of the Sun: A Summary of the Results from NASA's Solar Maximum Mission. Berlin: Springer, p. 301.

Chen H., Zhang J., Ma S., et al., 2015. Astrohys. J., vol. 808, p. L24.

Cheung M.C.M., Isobe H., 2014. Living Rev. Solar Phys., vol. 11, article id. 3.

Cowling T.G., 1957. Magnetohydrodynamics. London: Interscience Publ., Great Britain.

Dalmasse K., Aulanier G., Demoulin P., Kliem B., Török T., Pariat E., 2015. Astrophys. J., vol. 810, article id. 17.

Fursyak Yu.A., 2018. Geomagnetism and Aeronomy, vol. 58, pp. 1129-1135.

Fursyak Yu.A., Kutsenko A.S., Abramenko V.I., 2020. Solar Phys., vol. 295, article id. 19.

Galeev A.A., Rosner R., Serio S., Vaiana G.S., 1981. Astrophys. J., vol. 243, pp. 301-308.

Georgoulis M.K., Titov V.S., Mikic Z., 2012. Astrophys. J., vol. 761, article id. 61.

Gosain S., Demoulin P., Lopez Fuentes M., 2014. Astrophys. J., vol. 793, article id. 15.

Heyvaerts J., Priest E.R., Rust D.M., 1977. Astrophys. J., vol. 216, pp. 123-137.

Hoeksema J.T., Liu Y., Hayashi K., et al. 2014. Solar Phys., vol. 289, pp. 3483-3530.

Hollweg J.V., 1984. Astrophys. J., vol. 277, pp. 392-403.

Holman G.D., 1985. Astrophys. J., vol. 293, pp. 584-594.

Ionson J., 1984. Astrophys. J., vol. 276, pp. 357-368.

Jain K., Tripathy S.C., Hill F., 2017. Astrophys. J., vol. 849, article id. 94.

Jiang, C., Wu, S.T., Yurchyshyn, V., 2016. Astrophys. J., vol. 828 , article id. 62 .

Khodachenko M., Haerendel G., Rucker H.O., 2003. Astron. Asrophys., vol. 401, pp. 721-732.

Khodachenko M.L., Zaitsev V.V., 2002. Astrophys. Space. Sci., vol. 279, pp. 389-410. 
Kontogiannis I., Georgoulis M.K., Park S.-H., Guerra J.A., 2017. Solar Phys., vol. 292, article id. 159.

Leka K.D., Canfield R.C., McClymont A.N., van DrielGesztelyi L., 1996. Astrophys. J., vol. 462, pp. 547-560.

Lemen J.R., Title A.M., Akin D.J., et al., 2012. Solar Phys., vol. 275, pp. 17-40.

Li Y., Xue J.C., Ding M.D., et al., 2018. Astrophys. J. Lett., vol. 853, p. L15.

Liu Y., Sun X., Török T., Titov V.S., Leake J.E., 2017. Astrophys. J., vol. 846, p. L6.

Longcope D.W., Welsch B.T., 2000. Astrophys. J., vol. 545, pp. 1089-1100.

McClymont A.N., Fisher G.H., 1989. Washington DC American Geophysical Union Geophysical Monograph Series, vol. 54, pp. 219-225.

McMaken T.C., Petrie G.J.D., 2017. Astrophys. J., vol. 840, article id. 100.

Melrose D.B., 1991. Astrophys. J., vol. 381, pp. 306-312.

Melrose D.B., 1995. Astrophys. J., vol. 451, pp. 391-401.

Melrose D.B., Dulk G.A., 1984. Astrophys. J., vol. 282, pp. 308-315.

Morgan H., Pickering J., 2019. Solar Phys., vol. 294, article id. 135.

Parker E.N., 1973. Astrophys. J., vol. 180, pp. 247-252.

Parker E.N., 1988. Astrophys. J., vol. 330, pp. 474-479.

Parker E.N., 1996. Astrophys. J., vol. 471, pp. 485-496.

Priest E.R., Forbes T.G., 2002. Astron. Astrophys. Rev., vol. 10, pp. 313-377.

Pesnell W.D., Thompson B.J., Chamberlin P.C., 2012. Solar Phys., vol. 275, pp. 3-15.

Reale F., Peres G., 2000. Astrophys. J., vol. 528, p. L45.
Scherrer P.H., Schou J., Bush R.I., et al., 2012. Sol. Phys., vol. 275, pp. 207-227.

Severny A.B., 1964. Space Sci. Rev., vol. 3, pp. 451-486.

Sokolov V.S., Kosovichev A.G., 1978. Solar Phys., vol. 57, pp. 73-79.

Spicer D.C., 1991. In Ulmschneider P., et al. (Eds), Mechanisms of Chromospheric and Coronal Heating. Berlin: Springer-Verlag, p. 547.

Sprangler S.R., 2009. Nonlin. Processes Geophys., vol. 16, pp. 443-452.

Stenflo J.O., 1969., Solar Phys., vol. 8, pp. 115-118.

Stepanov A.V., Zaitsev V.V., Nakariakov V.M., 2012. Coronal Seismology. Wienheim: WILEY-VCH, Germany.

Sui L., Holman G.D., 2003. Astrophys. J., vol. 596, p. L251.

Sun X., Bobra M.G., Hoeksema J.T., et al., 2015. Astrophys. J. Lett., vol. 804, p. L28.

Thalmann J.K., Su Y., Temmer M., Veronig A.M., 2015. Astrophys. J. Lett., vol. 801, p. L23.

Török T., Kliem B., 2003. Astron. Astrophys., vol. 406, pp. 1043-1059.

Török T., Leake J.E., Titov V.S., et al., 2014. Astrophys. J., vol. 782, p. L10.

Zaitsev V.V., Khodachenko M.L., 1997. Radiophys. Quantum Electron., vol. 40, pp. 114-138.

Zaitsev V.V., Kronshtadtov P.V., 2016. Radiophys. Quantum Electron., vol. 59, pp. 169-176.

Zaitsev V.V., Shibasaki K., 2005. Astronomy Reports, vol. 49, pp. 1009-1017.

Zaitsev V.V., Stepanov A.V., 2008. Uspekhi Fiz. Nauk, vol. 51, pp. 1123-1160.

Zaitsev V.V., Stepanov A.V., Urpo S., Pohjolainen S., 1998. Astron. Astrophys., vol. 337, pp. 887-896. 